\title{
Measurement and Evaluation of the Autonomic Nervous Function in Daily Life
}

\author{
Makoto Komazawa',2, Kenichi Itao ${ }^{2,3}$, Hiroyuki Kobayashi' ${ }^{3}$, Zhiwei Luo ${ }^{4}$ \\ ${ }^{1}$ Graduate School of System Informatics, Kobe University, Kobe, Japan \\ ${ }^{2}$ WINFrontier Co. Ltd., Tokyo, Japan \\ ${ }^{3}$ JUNTENDO University, Tokyo, Japan \\ ${ }^{4}$ Organization of Advanced Science and Technology Kobe University, Kobe, Japan \\ Email: makoto.komazawa@winfrontier.com
}

Received 15 June 2016; accepted 12 July 2016; published 15 July 2016

Copyright (C) 2016 by authors and Scientific Research Publishing Inc.

This work is licensed under the Creative Commons Attribution International License (CC BY).

http://creativecommons.org/licenses/by/4.0/

(c) (†) Open Access

\begin{abstract}
We developed a smart-phone based system to measure the activities of autonomic nervous system during everyday life. Using commonly marketed smart phones, by touching your fingertips on the phone's camera over a short time of about 30 seconds, it will detect changes in the brightness of the blood flow and in turn analyze your heart rate variability. By using this system, about 100,000 cases were measured and from this large amount of data regarding heart rate variability, we evaluated the autonomic nervous function in their daily life. As a result, for the correlation between autonomic nervous system and age, we found that as the increase of age, the total power becomes decreased and the sympathetic nervous system tends to increase between thirties and fifties. For the correlation between autonomic nervous system and BMI (Body Mass Index), it is found that in general, the higher the BMI, the lower the total power and the stronger the sympathetic nervous system. In other words, people who are fat are lower about the total power and stronger about the sympathetic nervous system. In addition, for the correlation between autonomic nervous system and one day life, it is found that total power and sympathetic function tend to increase, while as evening approaches, sympathetic function tends to become suppressed.
\end{abstract}

\section{Keywords}

Heart Rate Variability Analysis, Autonomic Nervous System, Age, BMI

\section{Introduction}

It is extremely important to check health conditions scientifically in daily life as Japan is facing a super-aging 
society problem. For which, we need to consider our health during living at home, city, and/or workplaces under all kinds of conditions of the weather, gender, aging, et al., but not only dependent on hospital. It is clear that, when people are continuously subjected to large amounts of stress for long periods of time throughout their everyday lives, it will cause abnormalities of their autonomic nervous systems [1]. For this reason, in our study, we focus on establishing a user-friendly measuring technology for people to use every time and everywhere [2]. More specifically, this system uses smart phones and detects changes in pulses from variations in skin color when users are pushing their fingertip firmly against the camera of the device for about 30 seconds.

This system has been released as an iPhone application from Apple Inc. [3] as well as an Android application from Google Inc. [4] for free. Over a million people have downloaded it (as of May, 2016). In addition, from a point of view of public health, it is important to make comparative analyses on the distribution of health of people with different attributes at different times and locations. This research established technology to collect, store, and process big data related to health and used a large quantity of the data about the autonomic nervous system to evaluate the health in our daily lives.

In a previous study, researchers used special pulse wave sensors and small heart rate sensors and reported that the total power — the amount of activity—of the autonomic nervous system decreases with age [5]-[7], and that the higher the BMI (body mass index), the lower its total power [8]. However, there were only several hundred subjects at most for the study. In addition, another previous study that looked at BMI and total power [8] only looked at women suffering from menopausal disorders, and like the other study, there are no examples of analyses with a large amount of big data on the autonomic nervous system with a general selection of subjects. Similarly, there are no examples to analyze the relationship between the autonomic nervous system and circadian variation. This study will also report the results of analyses on the following three subjects based on a large amount of data on the autonomic nervous system from approximately 100,000 cases that were measured using our system:

1) CORRELATION BETWEEN THE AUTONOMIC FUNCTION AND AGE.

2) CORRELATION BETWEEN THE AUTONOMIC FUNCTION AND BMI.

3) CORRELATION BETWEEN THE AUTONOMIC FUNCTION AND CIRCADIAN VARIATION.

\section{Measurement System of the Autonomic Nervous System}

\subsection{Overview and Measurement Principles of the System}

Overview of the system we developed is shown in Figure 1. This is a client-server system which includes a

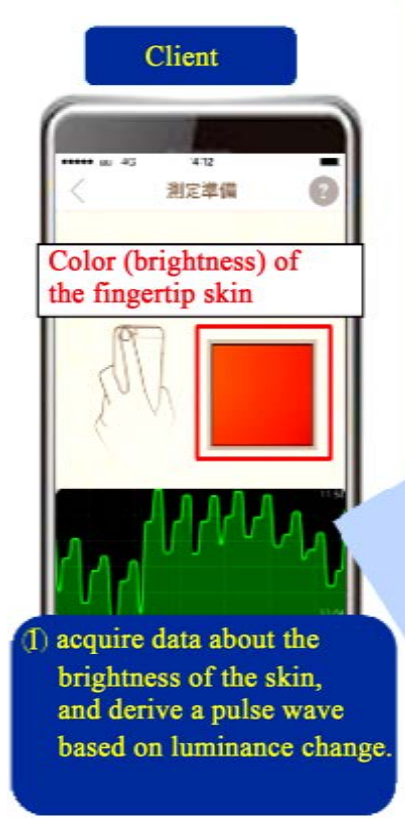

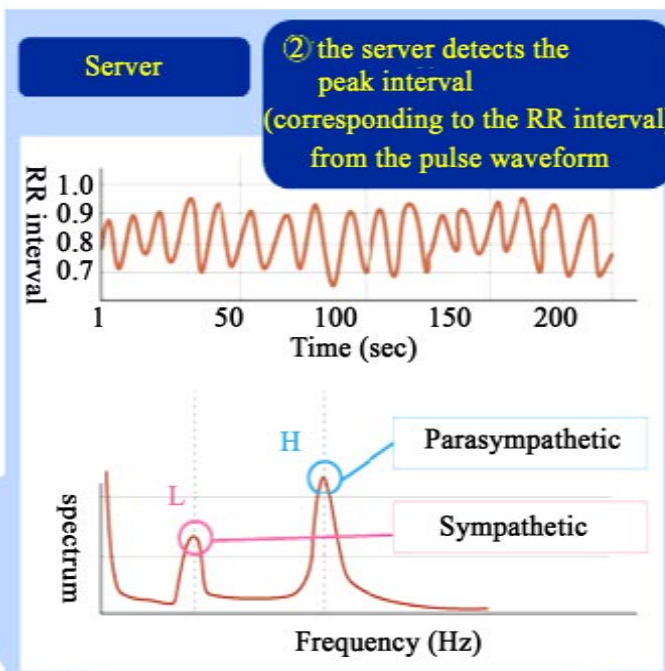

(3) calculates the sympathetic-para sympathetic activity Indicator

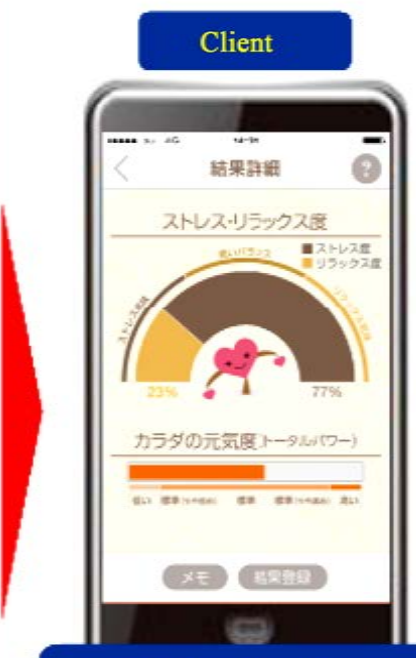

(4) visualize on the client terminal smart-phone.

Figure 1. Overview of system development. 
smart phone as the client terminal, and a server for analysis processing. The system is being built using Java and Objective-C.

Each time when humans breathe, the amount of hemoglobin contained in the blood rises and falls, and as a consequence, the color (brightness) of the fingertip skin changes subtly [9]. Therefore, by using a smart phone and applying a finger to the camera part, it is possible to continuously acquire data about the brightness of the skin and derive a pulse wave based on luminance change. On the other hand, the server detects the peak interval (corresponding to the RR interval) from the pulse waveform. Frequency analysis is applied to the fluctuation of the obtained RR interval, which in turn be used to calculate the sympathetic-parasympathetic activity indicator, as the autonomic nervous index. Finally, the autonomic nervous index is analyzed and processed by the server and be visualized on the client terminal smart-phone.

\subsection{Analysis Processing}

The flow of analysis in the system is shown in Figure 2. This system provides largely two types of analysis processing: "1. Luminance waveform analysis engine" and "2. Autonomic nervous system analysis engine". Firstly, for the step of "1-1 Noise removal filter of luminance data" in the "1. Luminance waveform analysis engine", since the influence of the high-frequency component of the noise is large, waveform data smoothing is applied using the "Savitzky-Golay method [14]" to the luminance data acquired from the smart-phone camera. For the smoothing of the data, the optimum value for the width of the filter window is determined using a secondary differential value, by simulating with a program the value when the waveform is most stable. In " $1-2$ Peak Detection Algorithm”, peaks are detected by sign inversion from the smoothed luminance waveform, and RR interval waveform (peak interval waveform) is obtained.

Next, in the step of "2-1 Outlier removal logic of RR interval" in "2. Autonomic nervous system analysis engine”, values are filtered by the difference from the average value of the most recent RR interval, RR interval value that exceeds a certain thresholds is estimated as an outlier value. Furthermore, a low pass filter is applied to the obtained RR interval waveform ("2-2 Low-pass filter"), high frequency noise is removed, frequency analysis is performed on the RR interval waveform in "2-3 Frequency Analysis Algorithm", and the autonomic index is calculated. The calculation method of the autonomic nervous index conforms to the procedures of the paper [10].

(A series of analysis algorithms are patent pending.) A supplementary explanation of the processing is given below.

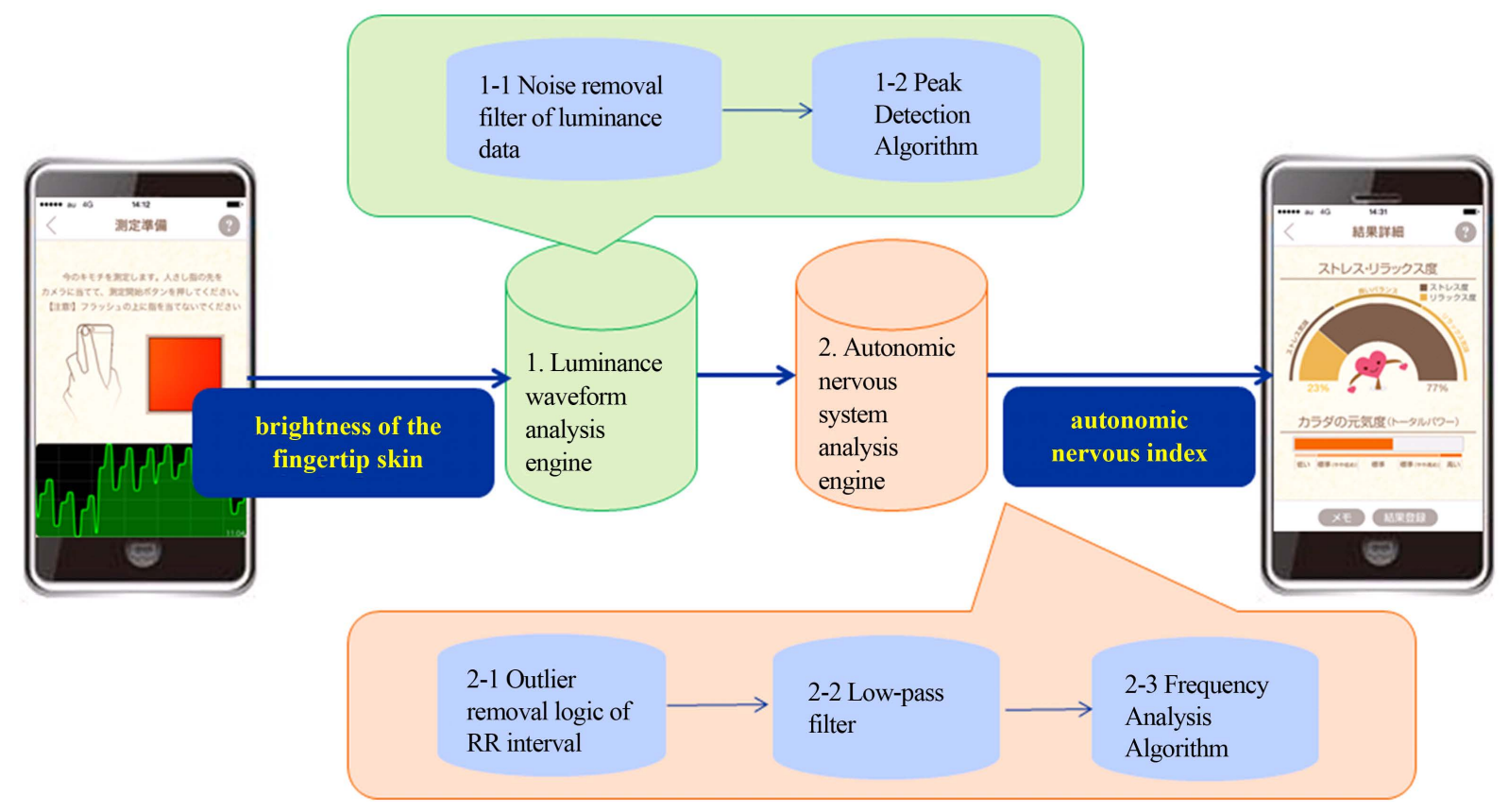

Figure 2. Analysis processing flow. 
[1-2 Peak Detection Algorithm]

The RR interval waveform (peak interval waveform) is acquired by estimating the peaks to be the points upon the smoothed luminance waveform at which the value of the first order differential is zero, and the value of the second order differential becomes negative.

[2-1 Outlier removal logic of RR interval]

[STEP1]

Calculate mean $\mathrm{HR}(X)$ as the moving average of $X$ heartbeats when the heart rate HR satisfies equation (1)

$$
|\operatorname{HR}(n)-\operatorname{HR}(n-1)| \leq Y
$$

where

$\operatorname{HR}(n)$ is the nth heart rate,

Mean HR $(X)$ is the moving average of the heart rate HR of $X$ heartbeats.

[STEP2]

$$
\mid \text { mean } \operatorname{HR}(X)-\operatorname{HR}(n) \mid \geq Z
$$

A heart rate (its corresponding RR interval) that exceeds the range of equation (2) is excluded as an error value. In this study, the variables are set at $X=8, Y=15$, and $Z=30$.

These parameters conform to values used in the authors' previous studies [6] [7].

[2-3 Frequency Analysis Algorithm]

In general, fluctuations occur in the heart rate of living bodies, and peaks can be seen at certain frequencies in a spectral analysis of the RR interval waveform. In humans, a high frequency component $(0.15 \mathrm{~Hz}-0.40 \mathrm{~Hz}$ : $\mathrm{HF})$, which represents variations in the respiratory cycle, and a low frequency component $(0.05 \mathrm{~Hz}-0.15 \mathrm{~Hz}$ : LF), which reflects fluctuations in blood pressure, are seen, with both of these reflecting autonomic nervous activity. HF is believed to respond to parasympathetic innervation, while LF responds to both sympathetic and parasympathetic innervation, and LF/HF reflects sympathetic function [10].

\subsection{Precision of Analyzed Value}

In order to verify the accuracy of the heart rate to check the variability analysis system using a smart-phone camera developed in this study, precision comparison was applied using dedicated fingertip pulse wave sensor system [11] (WIN Frontier Co., Ltd.). Measurement time with the fingertip pulse wave sensor and smart-phone camera was set to 38 seconds (using MEM for frequency analysis technique) by 11 subjects of men and women with their ages from 21 to 60 years old. Totally, 261 set of data were measured. While the subjects were sitting in a resting state, the index finger of one hand was equipped with a dedicated fingertip pulse wave sensor, and simultaneous measurement was carried out also on the index finger of the other hand by touching it to the smartphone camera.

The correlation diagram of the autonomic balance (ratio of the sympathetic and parasympathetic nervous systems), when the simultaneous measurements were carried out with a fingertip pulse wave sensor and smartphone camera, is shown in Figure 3. The correlation coefficient is 0.8, significant positive correlation was observed when subjected to a significant difference test $(\mathrm{p}<0.05)$.

In addition, the correlation diagram of the amount of activity of the autonomic nervous system, which is the sum of the sympathetic and parasympathetic systems, is shown in Figure 4. The correlation coefficient is 0.89 , significant positive correlation was observed when subjected to a significant difference test $(\mathrm{p}<0.05)$.

Finally, Figure 5 shows the correlation diagram of the heart rate. The correlation coefficient is 0.99 , significant positive correlation was observed when subjected to a significant difference test ( $\mathrm{p}<0.05)$.

It is then clear that our system can realize the similar precision of the measurement compared with that of the fingertip pulse wave sensor. Moreover, our system can be easily used to obtain the tendencies of the autonomic nervous state during everyday life.

\section{Measurement Data Analysis}

\subsection{Methods}

Here, we analyzed autonomic data measured by heart rate variability analysis system [2] with a smart-phone 


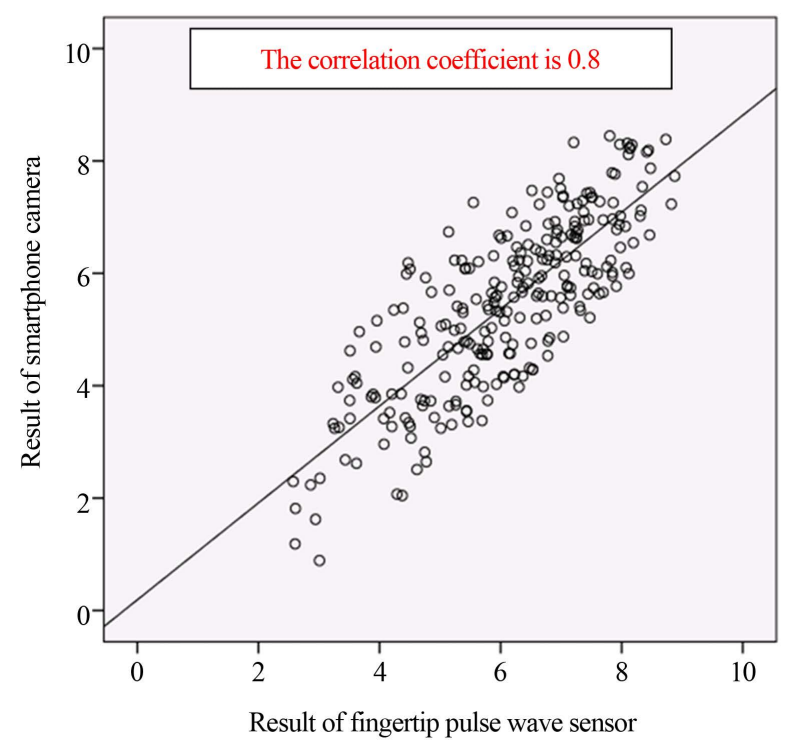

Figure 3. Correlation diagram of the autonomic balance.

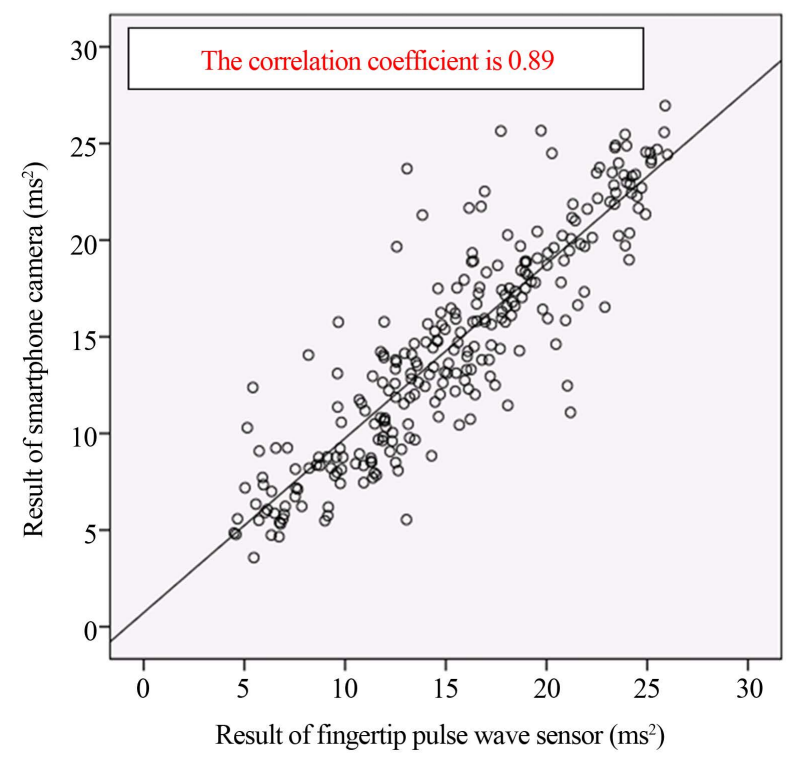

Figure 4. Correlation diagram of the amount of activity of the autonomic nervous system (total power).

camera. Frequency analysis technique is in accordance with the procedure of the paper [10], $0.04 \mathrm{~Hz}-0.15 \mathrm{~Hz}$ is calculated as low-frequency components (LF), $0.15 \mathrm{~Hz}-0.4 \mathrm{~Hz}$ as high-frequency component (HF).

The value of $\mathrm{LF} / \mathrm{HF}$ is used as an indicator of the sympathetic nervous function, as an indicator of tension, excitement, stress [10].

In addition, the sum of the LF and HF is called total power (TP), it is an index representing the activity of the autonomic nervous system [10]. The total power is correlated with fatigue, the smaller the value, the less fatigue is stored [12].

In this study we performed analysis based on the autonomic nervous system data of 27,307 people (male: 6394, female: 20,913). Regarding the use of the measurement data, we have the consent of the measured person, based on the Code of Ethics of WIN Frontier Corporation.

In Table 1, the age and sex of the participants is shown. Statistical processing for this study was done using the IBM SPSS Statics Version 22. Also, the significance level of the test was 5\%. 


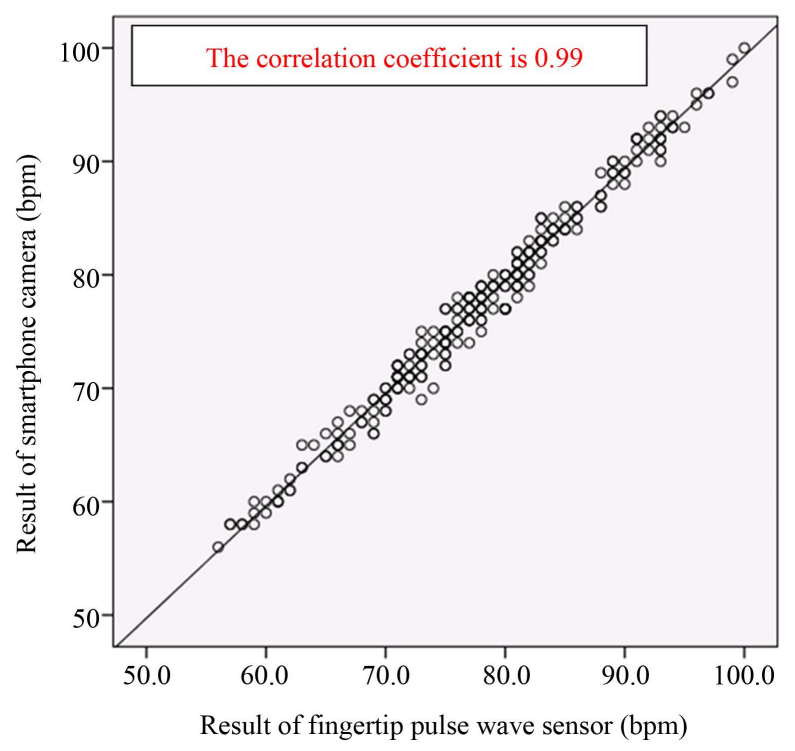

Figure 5. Correlation diagram of the heart rate.

Table 1. Age and gender of participants.

\begin{tabular}{cccc}
\hline Age & Man & Female & Total \\
\hline 10 's & 1058 & 9448 & 10,506 \\
20’s & 1433 & 7621 & 9054 \\
30’s & 1342 & 1856 & 3198 \\
40 's & 1406 & 1404 & 2810 \\
50’s & 884 & 528 & 1412 \\
Over 60’s & 271 & 56 & 327 \\
Total & 6394 & 20,913 & 27,307 \\
\hline
\end{tabular}

\subsection{Correlation between the Autonomic Function and Age}

In this section, we investigated the relationship between autonomic function and age. The contents of the measurement data used in this analysis are shown in Table 2.

When the logarithm was taken for the total power, the activity index of the autonomic nervous system that normality increases [5], total power of each measurement data was log transformed (LnTP), and regression analysis was performed with the age. Figure 6 shows the scatter diagram of the total power and age. As a results of the regression analysis, significant negative correlation was observed between the total power and age $(\mathrm{p}<$ $0.01)$.

Then, we classified them in six groups starting from teens to $60 \mathrm{~s}$ and over, and in order to investigate the difference between groups, multiple comparisons were performed using the Games-Howell method [15]. The results are shown in Figure 7.

As a result, as the age of the group increases, the total power was reduced significantly. This trend was also seen similarly in the case of both genders.

In previous studies of [5]-[7], a tendency of total power was observed decreasing as age increases, a similar tendency was also confirmed in this study. In this way, the results suggest that with the increase of age, the functional amount of activity of the autonomic nervous system decreases.

Then, the correlation between the LF/HF and age has been examined, as an indicator of the sympathetic nervous function. When the logarithm was taken of the LF/HF of each measurement data, since it is normality increases [5], the LF/HF of each measurement data was log transformed (LnLF/HF), and multiple comparisons were made using the Games-Howell method [15]. 


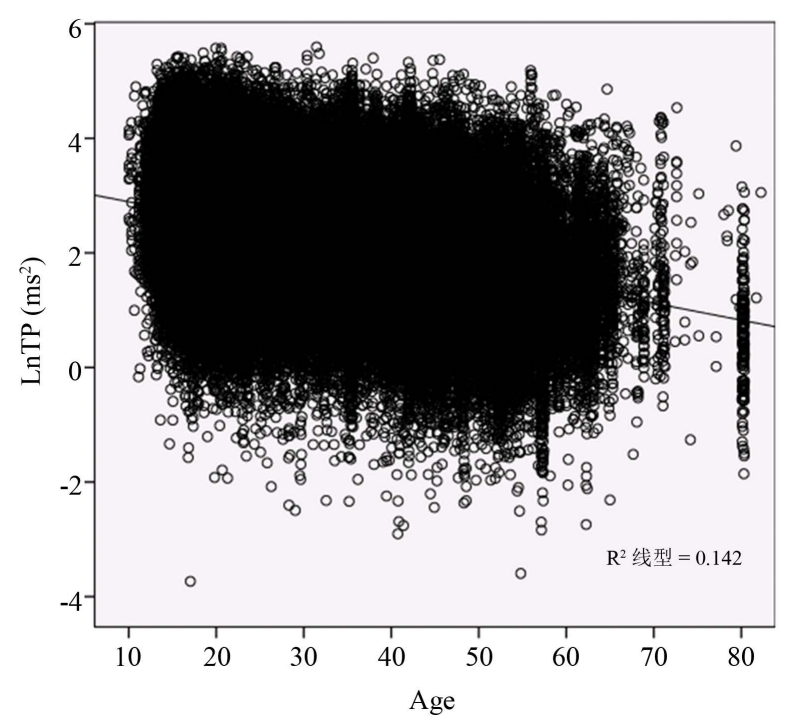

Figure 6. Scatter diagram of LnTP and age.

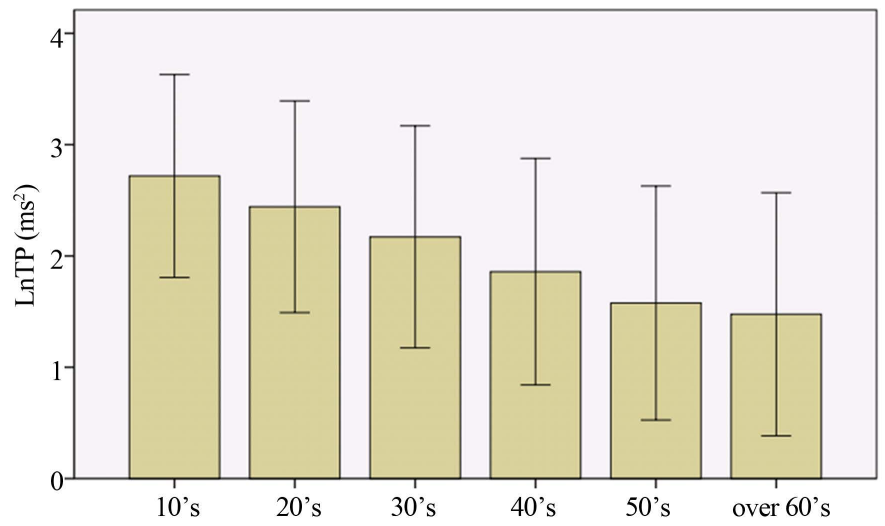

Figure 7. Correlation between LnTP and age.

Table 2. Number of measurement data for each age and gender.

\begin{tabular}{cccc}
\hline Age & Man & Female & Total \\
\hline 10 's & 3977 & 33,047 & 37,024 \\
20’s & 5795 & 30,449 & 36,244 \\
30’s & 9379 & 11,759 & 21,138 \\
40’s & 9459 & 12,194 & 21,653 \\
50’s & 7671 & 4717 & 12,388 \\
Over 60’s & 1749 & 452 & 2201 \\
Total & 38,030 & 92,618 & 130,648 \\
\hline
\end{tabular}

The results are shown in Figure 8. As a result, a significantly higher tendency of the sympathetic nervous function has been observed between the ages of 30 - 50 s. In previous studies, Zhang et al. have investigated about LF/HF and aging. Their results show an inverted U-shaped pattern with a peak at around age $50 \mathrm{~s}$ [13], in the results of this study, a similar tendency of an inverted U-shaped pattern was observed. In terms of gender, the peak value of the LF/HF for men was in their $40 \mathrm{~s}$, for women, it tended to be in their $50 \mathrm{~s}$.

The increase of sympathetic nerve function in the ages of $30-50 \mathrm{~s}$ is thought to be caused by a variety of 


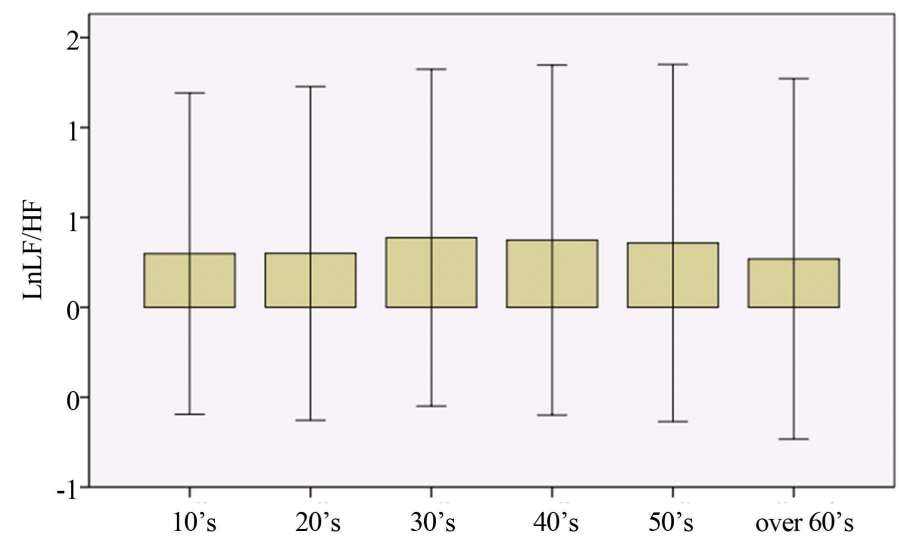

Figure 8. Correlation between LnLF/HF and age.

factors, not only physiological aging changes, but also a possibility that environmental factors are significantly involved.

More than young people in 20, in the case of people in their 30 - 50 s, with marriage, childbirth and work, their social responsibility increases. This is the period of big environmental changes. Such environmental influences are also considered likely to affect the autonomic nervous system.

On the other hand, the reason that sympathetic nerve function is suppressed in people in their $60 \mathrm{~s}$ or over can be attributed to many people celebrating their age of retirement, that the stress from work has been reduced, this is a generation that maintains their peace of mind.

\subsection{Correlation between the Autonomic Function and BMI}

In this section, we investigated the correlation between total power and BMI. The BMI is ratio of body weight to the square of the height. It is a body mass index that represents the degree of obesity of people. In addition, according to the criteria of the paper [14], the degree of obesity is calculated from the BMI. The correlation between BMI and obesity is shown in Table 3.

The obesity index of the subjects used in this analysis and the contents of the measurement data are shown in Table 4 and Table 5. Then, the degree of obesity calculated from the BMI is classified into four groups from low weight to obese (2 degrees or more), in order to investigate the difference between groups, the TP of each measurement data were log transformed (LnTP), and subjected to multiple comparison by the Games-Howell method [15]. The results are shown in Figure 9. The result shows that as the BMI increases, the total power has tended to decrease significantly. This trend was observed similarly in both genders. (However, for women only, there is no significant difference between low weight and the standard.)

In previous studies that examined the BMI and total power [8], where the subjects were only women who were suffering from menopause, a tendency was observed that, the higher the BMI, the more decreases the total power. In this study, a similar tendency could be confirmed with general subjects. In this way, the results suggest that the more obese a person is, the more the amount of activity function of the autonomic nervous system decreases.

Then, the correlation between the LF/HF, which is an indicator of sympathetic function, and BMI was examined. The LF/HF of each measurement data was log transformed (LnLF/HF), and they were subjected to multiple comparison by the Games-Howell method [15]. The results are shown in Figure 10. As a result, compared with low weight, in the case of obesity (degree 1), obesity (degree 2 or above), significantly increasing tendency has been observed in the sympathetic nerve function. This trend was seen similarly in both genders. As the sympathetic system is activated, blood pressure rises, it is said that in the case of obese people there is a tendency for sympathetic systems to activate easily [15]. In this study, the results suggest a similar tendency.

\subsection{Correlation between the Autonomic Function and Circadian Variation}

In this section, the correlation between autonomic function and circadian variation was examined. Here each day was separated into eight sections of three hours each, and they were subjected to analysis. The contents of the 


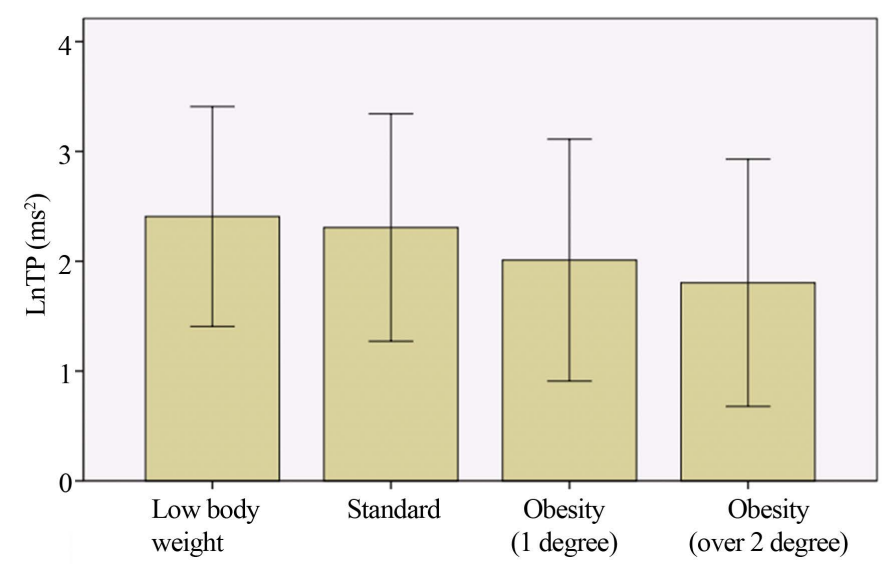

Figure 9. Correlation between LnTP and obesity.

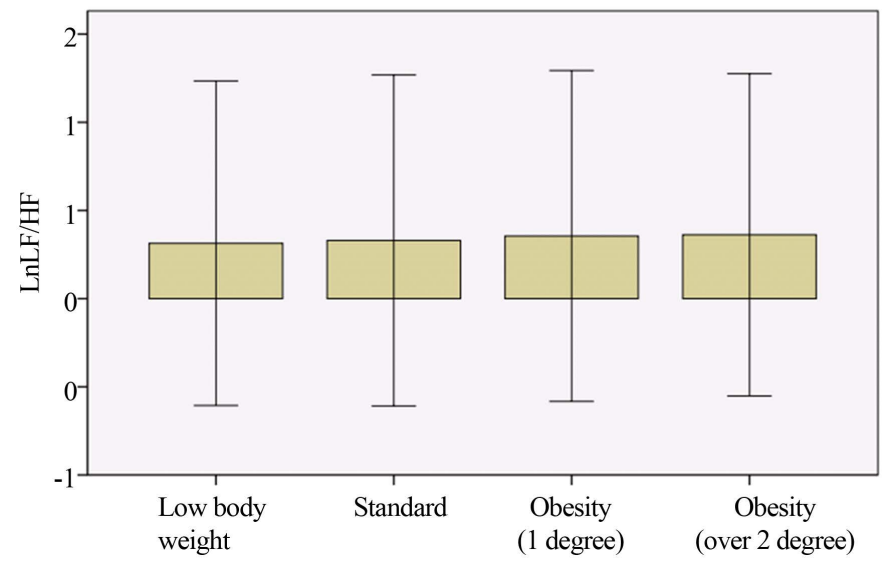

Figure 10. Correlation between LnLF/HF and obesity.

Table 3. Correlation between BMI and obesity.

\begin{tabular}{cc}
\hline BMI & Degree of obesity \\
\hline Under 18.5 & Low body weight \\
$18.5-25$ & Standard \\
$25-30$ & Obesity (1 degree) \\
$30-35$ & Obesity (2 degree) \\
$35-40$ & Obesity (3 degree) \\
Over 40 & Obesity (4 degree) \\
\hline
\end{tabular}

Table 4. Degree of obesity of subjects.

\begin{tabular}{cccc}
\hline Degree of obesity & Man & Female & Total \\
\hline Low body weight & 407 & 3073 & 3480 \\
Standard & 4662 & 16,249 & 20,911 \\
Obesity (1 degree) & 1049 & 1232 & 2281 \\
Obesity (over 2 degree) & 276 & 359 & 635 \\
Total & 6394 & 20,913 & 27,307 \\
\hline
\end{tabular}


Table 5. The number of measured data for different degrees of obesity.

\begin{tabular}{cccc}
\hline Degree of obesity & Man & Female & Total \\
\hline Low body weight & 1741 & 13,010 & 14,751 \\
Standard & 29,687 & 71,984 & 101,671 \\
Obesity (1 degree) & 5468 & 5803 & 11,271 \\
Obesity (over 2 degree) & 1134 & 1821 & 2955 \\
Total & 38,030 & 92,618 & 130,648 \\
\hline
\end{tabular}

Table 6. The number of measured data for different times of day.

\begin{tabular}{rccc}
\hline Time zone & Man & Female & Total \\
\hline 0-3 o'clock & 3428 & 10,617 & 14,045 \\
3-6 o'clock & 1295 & 2949 & 4244 \\
6-9 o'clock & 4716 & 8653 & 13,369 \\
9-12 o'clock & 4267 & 9323 & 13,590 \\
12-15 o'clock & 5064 & 10,516 & 15,580 \\
$15-18$ o'clock & 4862 & 10,776 & 15,638 \\
$18-21$ o'clock & 6195 & 16,617 & 22,812 \\
$21-24$ o'clock & 8203 & 23,167 & 31,370 \\
Total & 38,030 & 92,618 & 130,648 \\
\hline
\end{tabular}

measured data used in this analysis are shown in Table 6. The TP of the each measurement data were log transformed (LnTP), they were classified into eight groups of 3 hours each, in order to investigate the difference between groups, they were subjected to multiple comparison by the Games-Howell method [15].

The results are shown in Figure 11. As a result, in the early morning hours of 3 AM to 6 AM, a significant increase was observed in the tendency of the total power. This trend was seen similarly in both genders. In previous studies, regarding the circadian variation of autonomic function, it was observed that in the early morning before and after getting up, the total power of the autonomic nervous system has tended to increase [16], this is consistent with the results of the present study.

In addition, saliva cortisol, which is said to correlate with adrenal fatigue, is said to show circadian variation as it has the highest value early in the morning, and has the lowest value at night [16], a generally matching tendency has been found between the circadian variation of the total power of the autonomic nervous that is said to be correlated with fatigue [12].

Next, the LF/HF of each measurement data was log transformed (LnLF/HF), and they were subjected to multiple comparison by the Games-Howell method [15]. The results are shown in Figure 12.

As a result, it has been observed that early in the morning the sympathetic nerve function tends to be significantly increased, and as night comes on, the sympathetic function tends to be suppressed. This trend was seen similarly in both genders.

In previous studies, regarding the circadian variation of autonomic function, it was observed that sympathetic function becomes higher through the day from the morning, and over the evening, in reverse, the sympathetic nerve function tends to be suppressed, the results of the present study present a matching tendency.

\section{Conclusion}

We developed a system in this study that allows the measuring of the tendencies of the autonomic nervous system conveniently in our everyday life. Using commonly marketed smart phones, by touching your fingertips on your phone's camera over a short period of about 30 seconds, it will detect changes in the brightness of the blood flow and analyze your heart rate variability. When comparing with the heart rate variability analysis system using the dedicated fingertip pulse wave sensors, it shows that there is a correlation of approximately $80 \%$ 


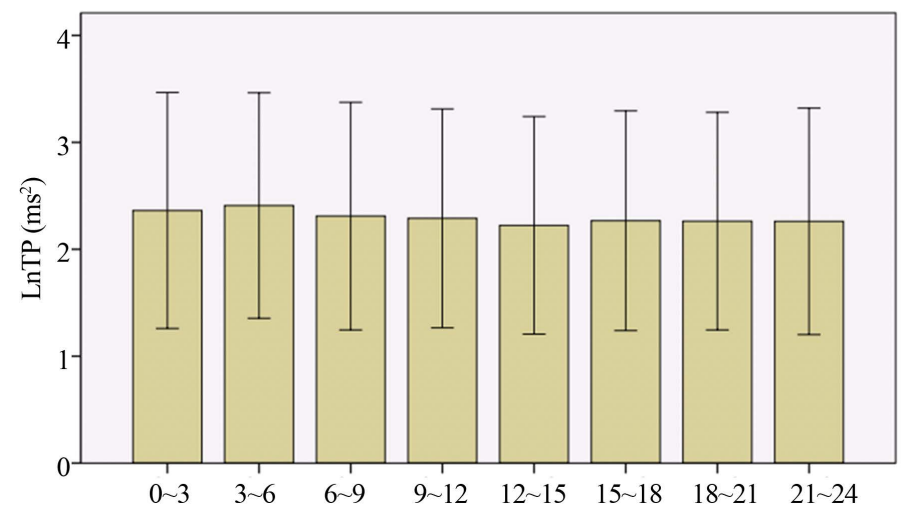

Figure 11. Correlation between LnTP and different times of day.

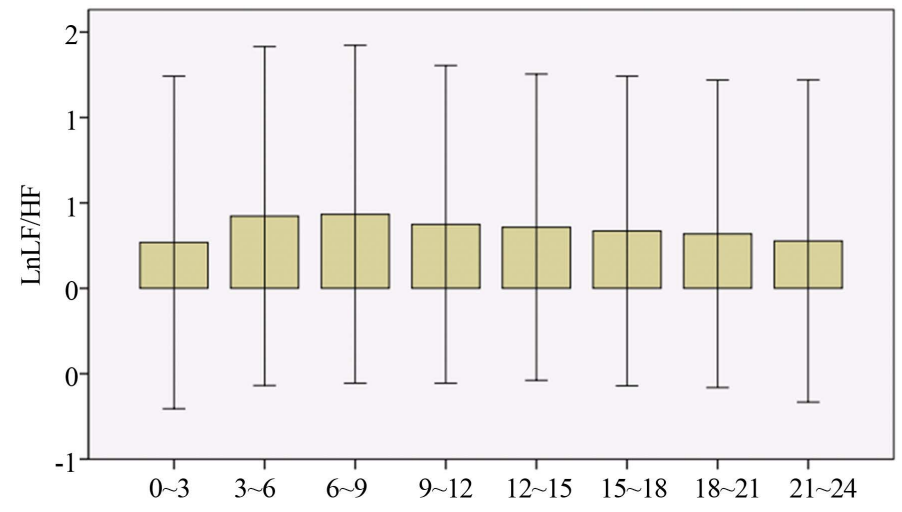

Figure 12. Correlation between LnLF/HF and different times of day.

[2]. Then, using this system, about 100,000 cases were measured and from this large amount of data regarding heart rate variability, we evaluated the autonomic nervous function in daily life. As a result, for the correlation between autonomic nervous system and age, we found that the more people age, the more they lose total power and that sympathetic nervous system tends to increase between their thirties and fifties. For the correlation between autonomic nervous system and BMI, we found that in general, the higher the BMI, the lower the total power and the stronger the sympathetic nervous system. In other words, people who are fat are lower about the total power and stronger about the sympathetic nervous system. For the correlation between autonomic nervous system and time of day, we found that total power and sympathetic function tend to increase at dawn. As evening approaches, sympathetic function tends to become suppressed. This is consistent with the diurnal variation in autonomic nervous function. Currently, by using the heart rate variability analysis system using a smart-phone camera [2], the autonomic system data of thousands of days are accumulated. Therefore, as future development, when the autonomic data has reached 1 million, we hope to continue the evaluation of daily autonomic function in more detail.

\section{References}

[1] Onaka, T. (2005) Stress and Its Neural Mechanisms. Journal of Pharmacological Sciences, 126, 170-173.

[2] Komazawa, M., Itao, K. and Luo, Z.W. (2015) Development of Heart Rate Variability Analysis System Using a SmartPhone Camera. 20th Symposium of Academy of Human Informatics, 19-20.

[3] WIN Frontier Co., Ltd. (2015) COCOLOLO_Check 8 Types of Feelings with the Fluctuation of the Heart Rate. https://itunes.apple.com/jp/app/cocololo-cafe-kimochishea/id973325431?mt=8\&ign-mpt=uo\%3D4

[4] WIN Frontier Co., Ltd. (2015) COCOLOLO-Heart Furnace-Stress Check Using the Fluctuation of Heart Rate. https://play.google.com/store/apps/details?id=com.winfrontier.COCOLOLO\&hl=ja

[5] Yukishita, T., Lee, K., Kim, S., Yumoto, Y., Kobayashi, A., Shirasawa, T. and Kobayashi, H. (2010) Age and Sex- 
Dependent Alterations in Heart Rate Variability Profiling the Characteristics of Men and Women in Their 30s. AntiAging Medicine, 7, 94-100. http://dx.doi.org/10.3793/jaam.7.94

[6] Itao, K., Komazawa, M., Katada, Y., Itao, K., Kobayashi, H. and Luo, Z.W. (2014) Age-Related Change of the Activity of Autonomic Nervous System Measured by Wearable Heart Rate Sensor for Long Period of Time: Pervasive Computing Paradigms for Mental Health. The Series Lecture Notes of the Institute for Computer Sciences, Social Informatics and Telecommunications Engineering, 100, 33-38. http://dx.doi.org/10.1007/978-3-319-11564-1_4

[7] Itao, K., Komazawa, M., Kobayashi, H. and Luo, Z.W. (2014) Correlation between Autonomic Function and Age in Japanese People Using the Analysis of Heart Rate Variability Data of 24 Hours. 19th Symposium of Academy of Human Informatics, 15.

[8] Moritani, T. (2009) The Role of Exercise and Nutrition in Menopausal Women. Menopause and Aging Health Care, 8, $12-20$.

[9] Toritani, A., et al. (2012) Reliable Pulse Detection Method by Portable Camera with a Measurement State Notification Function. Journal of Information Processing Society of Japan, Consumer Devices \& Systems, 2, 38-47.

[10] Task Force of the European Society of Cardiology and the North American Society of Pacing and Electrophysiology (1996) Heart Rate Variability: Standards of Measurement, Physiological Interpretation, and Clinical Use. Circulation, 93, 1043-1065. http://dx.doi.org/10.1161/01.CIR.93.5.1043

[11] WIN Frontier Co., Ltd. Lifescore Quick. http://www.winfrontier.com/lifescorequick.html

[12] Kuratsune, H., et al. (2011) Creation of Chronic Fatigue Diagnosis Guidelines and the Establishment of Objective Fatigue Diagnostic Methods for Patients Complaining of Chronic Fatigue Accompanied by Autonomic Abnormalities Health, Labor and Welfare Ministry Scientific Research, Disability Measures Research Department (Mental Disorders and Nerve and Muscle Disease Areas) 2009-2011. General Research Report, 1-114.

[13] Zhang, J. (2007) Effect of Age and Sex on Heart Rate Variability in Healthy Subjects. Journal of Manipulative and Physiological Therapeutics, 30, 374-379. http://dx.doi.org/10.1016/j.jmpt.2007.04.001

[14] Savitzky, A. and Golay, M.J.E. (1964) Smoothing and Differentiation of Data by Simplified Least Squares Procedures. Analytical Chemistry, 36, 1627-1639. http://dx.doi.org/10.1021/ac60214a047

[15] Games, P.A. and Howell, J.F. (1976) Pairwise Multiple Comparison Procedures with Unequal N's and/or Variances: A Monte Carlo Study. Journal of Educational and Behavioral Statistics, 1, 113-125. http://dx.doi.org/10.3102/10769986001002113

[16] Shimizu, T. (2008) The Autonomic Function around the Clock. Japanese Society for Medical and Biological Engineering, 46, 154-159.

\section{Submit or recommend next manuscript to SCIRP and we will provide best service for you:}

Accepting pre-submission inquiries through Email, Facebook, Linkedin, Twitter, etc

A wide selection of journals (inclusive of 9 subjects, more than 200 journals)

Providing a 24-hour high-quality service

User-friendly online submission system

Fair and swift peer-review system

Efficient typesetting and proofreading procedure

Display of the result of downloads and visits, as well as the number of cited articles

Maximum dissemination of your research work

Submit your manuscript at: http://papersubmission.scirp.org/ 